\title{
The First Case Treated with Larotrectinib of a Novel TMTC2-NTRK3 Fusion Undifferentiated High-Grade Pleomorphic Sarcoma of the Chest
}

\section{Chujie Bai}

Peking University Cancer Hospital: Beijing Cancer Hospital

\section{Lu Zhang}

Peking University Cancer Hospital: Beijing Cancer Hospital

\section{Yaohui Wang}

The Beijing Water Conservancy Hospital

\section{Taiyan Guo}

Jiangsu Simcere Diagnostics Co.

\section{Xia You}

Jiangsu Simcere Diagnostics Co.

Zhengfu Fan ( $\square$ fanzhengfu05@163.com )

Peking University Cancer Hospital: Beijing Cancer Hospital

\section{Research Article}

Keywords: NTRK3, Larotrectinib, Undifferentiated high-grade pleomorphic sarcoma (UPS), Sarcoma

Posted Date: September 16th, 2021

DOl: https://doi.org/10.21203/rs.3.rs-843043/v1

License: (c) (i) This work is licensed under a Creative Commons Attribution 4.0 International License. Read Full License 


\section{Abstract}

Undifferentiated high-grade pleomorphic sarcoma (UPS) is a rare soft tissue sarcoma (STS) of mesenchymal origin, particularly the extremities and retroperitoneum, meanwhile it has been reported in almost all parts of the body. UPS is highly invasive and has a poor prognosis due to its clinical manifestations of painless mass and deep tumor site, which are usually found at an advanced stage. Patients with UPS tend to have a lower 5-year survival rate than patients with other types of STS. Recently, NTRK fusions were detected in many cancer types, such as thyroid cancer, colorectal cancer, non-small cell lung cancer, soft tissue tumors, uterine sarcomas, and melanomas. However, the mutation frequency of NTRK fusion in all cancers is only $0.1-1 \%$. Targeted therapy with NTRK inhibitors, such as Larotrectinib and Entrectinib, leads to a response in most patients with NTRK1/2/3 gene fusion-positive tumors. Herein, we present a 68-years old man diagnosed with stage IIIA (T2NOMOG3) UPS. Nextgeneration sequencing (NGS) revealed a novel TMTC2-NTRK3 fusion. The NTRK3 positivity was also detected by immunohistochemistry (IHC) and fluorescence in situ hybridization (FISH). He had a response to Larotrectinib. This report broadens the spectrum of NTRK fusions in UPS and highlights a new target for treatment.

\section{Background}

Undifferentiated high-grade pleomorphic sarcoma (UPS), originated from connective tissue, is a rare tumor, accounting for $2-5 \%$ of all primary malignant soft tissue sarcomas (STS) [1, 2]. UPS occurs most commonly in the extremities and retroperitoneum and has also been reported in other sites, such as in the left chest wall [3]. Metastasis occurs most frequently in the lung, bone, and liver. It often presents itself as a painless, enlarging, soft-tissue mass. Treatment consists of surgical excision and radiation.

The neurotrophic receptor tyrosine kinase (NTRK) genes, including NTRK1 (chromosome 1q21-q22), NTRK2 (chromosome 9q22), and NTRK3 (chromosome 15q25), encode a family of transmembrane receptor tyrosine kinase proteins, TrkA, TrkB, and TrkC. Trk respectively. These proteins are expressed in neuronal tissue and are involved in the development and maturation of the human central and peripheral nervous systems [4]. Molecular alterations (predominantly gene fusions) and aberrant activation of NTRK genes have been reported in multiple tumor types. Larotrectinib is the first highly selective oral tropomyosin receptor kinase (TRK) inhibitor approved by the Food and Drug Administration (FDA) for the treatment of adult and pediatric patients with advanced solid tumors harboring the NTRK gene fusion [57]. There are several ongoing clinical trials, which cover various types of malignancies, including UPS. Here, we first report a novel NTRK3 fusion of a UPS patient with a response of Larotrectinib. And we will review the works of literature associated with the UPS and clinical trials of NTRKs.

\section{Case Presentation}

A 69-year-old male patient presented to the hospital in May 2019 with a mass nodule on the left chest wall. Ultrasonography revealed the hypoechoic nodule measured $4.9 \mathrm{~cm} \star 1.5 \mathrm{~cm}$ in the subcutaneous 
muscle layer of the left chest wall. The postoperative pathological diagnosis was T2NOM0G3 (IIIA) Undifferentiated high-grade pleomorphic sarcoma (UHPS). Positron emission tomography-computed tomography (PET/CT) revealed several high metabolic lymph nodes in mediastinal station 3P. The patient received four cycles of postoperative radiotherapy (RT) of volumetric-modulated arc therapy (VMAT). After radiotherapy, the patient did not feel nausea, vomiting, abdominal pain, diarrhea, fever, exertion and leave the hospital. CT scans revealed multiple metastases in the lungs seven months after hospital discharge.

Next-generation sequencing (NGS) analysis based on a pan-cancer 539-gene panel was performed to discover potential therapeutic regimens. A novel TMTC2-NTRK3 fusion was detected in surgical tissue. The fusion contained exon1-exon9 of TMTC2 and exon15-exon36 of NTRK3 (Fig. 1a, b). Pan-TRK immunostaining and NTRK3 break-apart FISH demonstrated a strong positive in this case (Fig. 1c).

The patient was treated with Larotrectinib in July 2020. After three months of Larotrectinib therapy, no noticeable adverse reactions took place. Six months later of the Larotrectinib treatment, the patient had a stable disease though metastases in both lungs were enlarged than before (Fig. 2). Then the patient stopped Larotrectinib treatment.

\section{Discussion}

Undifferentiated pleomorphic sarcoma (UPS) was first described in 1961 by Kauffman as a histiocytic tumor with storiform growth in children [8]. UPS occurs most frequently in patients between 50 and 70 years [9], accounting for $28 \%$ of all soft-tissue sarcomas [10]. The major locations of UPS are the lower (49\%), upper extremities (19\%), while tumors of the abdominal cavity and retroperitoneum account for $16 \%$, and those of the trunk accounts for $9 \%$ [11]. UPS includes four histological types: storiformpleomorphic, giant cell, myxoid, and inflammatory subtypes. The UPS histological morphology varies; however, the classic form comprises spindle-shaped and round histiocytes arranged in a storiform pattern. A majority of UPS patients do not have typical clinical manifestations; so they present to the hospital late because of the relative absence of early clinical symptoms. If symptomatic, patients often suffer from abdominal discomfort, altered bowel motions, hard nodules, and occasionally weight loss, and so on. The tumor is often large at presentation since it usually does not pay attention for a long time.

The primary treatment of UPS is en-bloc resection of the tumor with free margins. However, the prognosis of UPS is extremely poor. Molecular studies suggest that biomarkers such as p-STAT3, SOCS3, MET, and KIT may be prognostic factors for UPS, correlated with different prognoses $[12,13]$. There are no effective chemotherapy options at present. The addition of RT to wide surgical excision for UPS has improved local control rates compared with surgery alone [14].

Recently clinical trial data and the subsequent accelerated FDA approval of the selective NTRK inhibitor, Larotrectinib, for treating adult and pediatric patients with solid tumors with NTRK fusion $[15,16]$. Larotrectinib has shown a dramatic and durable activity against locally advanced and metastatic solid tumors with NTRK fusions [17]. It is worth noting that this remarkable activity was seen regardless of 
tumor site of origin, histologic classification, or NTRK fusion type. Previous studies suggested that some cancer types, such as thyroid carcinoma, salivary gland carcinoma, infantile fibrosarcoma, are highly enriched for NTRK fusions $[18,19]$. NTRK fusions can be detected in $\varangle 5 \%$ non-GIST soft-tissue sarcomas. A retrospective analysis of 38,095 samples from 33,997 patients identified 87 patients with oncogenic NTRK1-3 fusions. NTRK fusions are seen in $0.68 \%$ of sarcoma and are seen in $17.7 \%$ of inflammatory myofibroblastic tumors [20]. Although most NTRK-fusion sarcomas occur in pediatric populations, they also affect a smaller number of adults. Thus far, some researchers have studied the incidence of NTRK3 fusion proteins in sarcoma; all of them are ETV6-NTRK3. In Ramamoorthy Nagasubramanian's paper which first reported an infantile fibrosarcoma patient with ETV6-NTRK3 fusion successfully treated with the tropomyosin-related kinase inhibitor LOXO-101 [21]. In the second study, Kenneth J. Caldwell reported that a newborn with ETV6-NTRK3 fusion-positive infantile fibrosarcoma was successfully treated with Larotrectinib [22]. While in the third paper [23], the patient was a child with ETV6-NTRK3 fusion-positive undifferentiated embryonal sarcoma of the kidney, successfully treated with adjuvant maintenance Larotrectinib therapy. However, the frequency of NTRK fusions, especially NTRK3 in UPS, and especially in Chinese UPS patients is not clear. So, it is the first time reported another NTRK3 fusion type, and it is a little different in the clinical response of Larotrectinib, which in our patient only got a stable disease (SD) result. Unresponsiveness to an NTRK inhibitor may be due either to the lack of an NTRK fusion or to the development of a resistance mechanism [24], such as acquired mutations in the kinase domain of the NTRK genes [25], including a mutation in NTRK3 that changes amino acid 623 in TRKC from glycine to arginine (G623R) or a similar mutation in the paralogous residue, G595R of NTRK1/TRKA. In the 2020 ASCO case report [26], they had discussed response and mechanisms of resistance to Larotrectinib and Selitrectinib in metastatic undifferentiated sarcoma harboring an oncogenic fusion of NTRK1, and they discovered the NTRK1 solvent-front mutation, which facilitates the tumor initially developed resistance to Larotrectinib. The gain-of-function KRAS G12V mutation was identified as a second mutation that might alter the immune microenvironment and affected TRK-inhibitor's function. However, our patient was detected with NTRK3 fusion and expressed NTRK3 kinase domain protein, and it is still not a good result when treated with Larotrectinib. We will focus on the patient's gained mutation in the future.

In our case, we identified a novel TMTC2-NTRK3 fusion-positive tumor in a Chinese UPS patient by DNASeq, IHC, and FISH, which expanded the NTRK fusion spectrum and reflected our clinical practice.

\section{Abbreviations}

UPS = Undifferentiated high-grade pleomorphic sarcoma, PET/CT = positron emission tomographycomputed tomography, VMAT = volumetric-modulated arc therapy, FISH = fluorescence in situ hybridization, IHC = immunohistochemistry, SMA = smooth muscle actin, STS = soft tissue sarcoma.

\section{Declarations}

Ethics approval and consent to participate This study conformed to the Declaration of Helsinki and was approved by the Ethics Committee of Peking University Cancer Hospital, China 
Data availability All data generated or analysed during this study are included in this published article.

Competing interests None.

Funding information None

Authors' contributions Chujie Bai and Lu Zhang conceived the idea, and is the patient's primary oncologist; Drafted the initial draft alongside comprehensive literature review by Taiyan Guo and Xia You; Additional details provided by Yaohui Wang assisted with material and data collection; Zhengfu Fan revised the draft before an additional round of revisions by all authors.

\section{Compliance with ethical standards}

\section{Disclosure of potential conflicts of interest None}

Consent for publication Written informed consent was obtained from the patient for the publication of this report and any accompanying images.

\section{References}

1. Peiper M, Zurakowski D, Knoefel WT, et al. Malignant fibrous histiocytoma of the extremities and trunk: an institutional review. Surgery.2004;135(1):59-66

2. Efe T, Heyse TJ, Schofer MD, et al. Malignant fibroushistiocytoma of the distal femur after an arthroscopic anterior cruciate ligament reconstruction: a case report and a review of the literature. BMC Cancer. 2010;10:264

3. Moradi A, Afsharfard A, Atqiaee K. Undifferentiated Pleomorphic Sarcoma Presenting as Abdominal Pain with a Pulsatile Mass. Case Rep Surg.2016;2016:8251043

4. Nakagawara A. Trk receptor tyrosine kinases: a bridge between cancer and neural development. Cancer Lett.2001;169(2):107-14

5. Drilon A et al. Efficacy of Larotrectinib in TRK Fusion-Positive Cancers in Adults and Children. N Engl J Med. 2018;378(8):731-739

6. Hyman DM et al. A Next-Generation TRK Kinase Inhibitor Overcomes Acquired Resistance to Prior TRK Kinase Inhibition in Patients with TRK Fusion-Positive Solid Tumors. J Clin Oncol. 2017;7(9):963-972

7. FDA. Larotrectinib Prescribing Information. Available from: https://www.accessdata.fda.gov/drugsatfda_docs/label/2018/211710s000lbl (2018)

8. Kauffman SL, Stout AP. Histiocytic tumors (fibrous xanthoma and histiocytoma) in children. Cancer. 1961;14:469-82

9. Hsiao PJ, Chen $\mathrm{GH}$, Chang $\mathrm{YH}$,et al. An unresectable retroperitoneal malignant fibrous histiocytoma: a case report. Oncol Lett. 2016;11(4):2403-2407

10. Cormier JN, Pollock RE. Soft tissue sarcomas. CA Cancer J Clin. 2004;54(2):94-109. 
11. Weiss SW, Enzinger FM. Malignant fibrous histiocytoma: an analysis of 200 cases. Cancer. 1978;41(6):2250-66

12. Pazzaglia L, Novello C, Conti A, Pollino S, Picci P, Benassi MS. miR-152 down-regulation is associated with MET up-regulation in leiomyosarcoma and undifferentiated pleomorphic sarcoma. Cell Oncol (Dordr). 2017;40(1):77-88

13. Bekki H, Kohashi K, Yamada Y, lura K, Ishii T, Maekawa A, et al. Phosphorylation of STAT3 in undifferentiated pleomorphic sarcoma is correlated with a favorable prognosis. Pathobiology. 2017;84(3):161-169

14. Tuan J, Vitolo V, Vischioni B, lannalfi A, Fiore MR, Fossati P, et al. Radiation therapy for retroperitoneal sarcoma. Radiol Med. 2014;119(10):790-802

15. Food and Drug Administration. FDA approves larotrectinib for solid tumors with NTRK gene fusions. FDA Drugs. 2018

16. Food and Drug Administration. FDA approves an oncology drug that targets a key genetic driver of cancer, rather than a specific type of tumor. FDA news release. 2018

17. Drilon A, Laetsch TW, Kummar S, et al. Efficacy of larotrectinib in TRK fusion-positive cancers in adults and children. N Engl J Med. 2018;378(8):731-739

18. Amatu A, Sartore-Bianchi A, Siena S. NTRK gene fusions as novel targets of cancer therapy across multiple tumour types. ESMO Open. 2016;1(2):e000023.

19. Knezevich SR, McFadden DE, Tao W, Lim JF, Sorensen PH. A novel ETV6-NTRK3 gene fusion in congenital fibrosarcoma. Nat Genet.1998;18(2):184-7

20. Solomon JP, Linkov I, Rosado A, et al. NTRK fusion detection across multiple assays and 33,997 cases: diagnostic implications and pitfalls. Mod Pathol. 2020;33(1):38-46

21. Nagasubramanian R, Wei J, Gordon P, Rastatter JC, Cox MC, Pappo A. Infantile Fibrosarcoma With NTRK3-ETV6 Fusion Successfully Treated With the Tropomyosin-Related Kinase Inhibitor LOXO101. Pediatr Blood Cancer. 2016;63(8):1468-70

22. Caldwell KJ, De La Cuesta E, Morin C, et al. A newborn with a large NTRK fusion positive infantile fibrosarcoma successfully treated with larotrectinib. Pediatr Blood Cancer. 2020;67(9):e28330

23. Carter-Febres M, Schneller N, Fair D, et al. Adjuvant Maintenance Larotrectinib Therapy in 2 Children With NTRK Fusion-positive High-grade Cancers. J Pediatr Hematol Oncol. 2020

24. Assi T, Rassy E, Nassereddine $H$, et al. TRK inhibition in soft tissue sarcomas: A comprehensive review. Semin Oncol. 2020;47(1):73-84

25. Siegel RL, Miller KD, Jemal A. Cancer statistics, 2019. CA Cancer J Clin. 2019;69: 7-34.

26. Hemming ML, Nathenson MJ, Lin JR, et al. Response and mechanisms of resistance to larotrectinib and selitrectinib in metastatic undifferentiated sarcoma harboring oncogenic fusion of NTRK1. JCO Precis Oncol. 2020; 4: 79-90

\section{Figures}


a

5

3'

$\mathbf{5}^{\prime}$

3

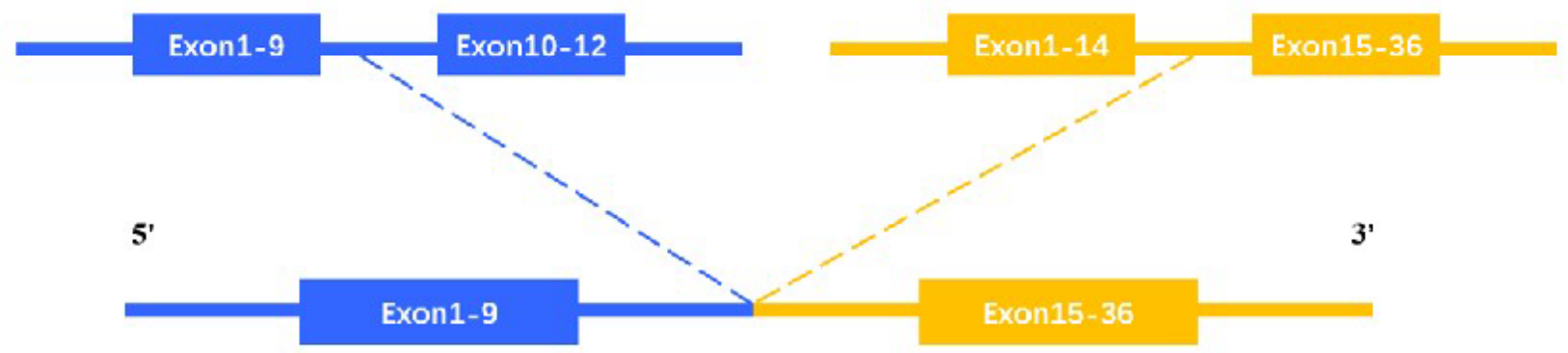

b

TMTC2-NTRK3

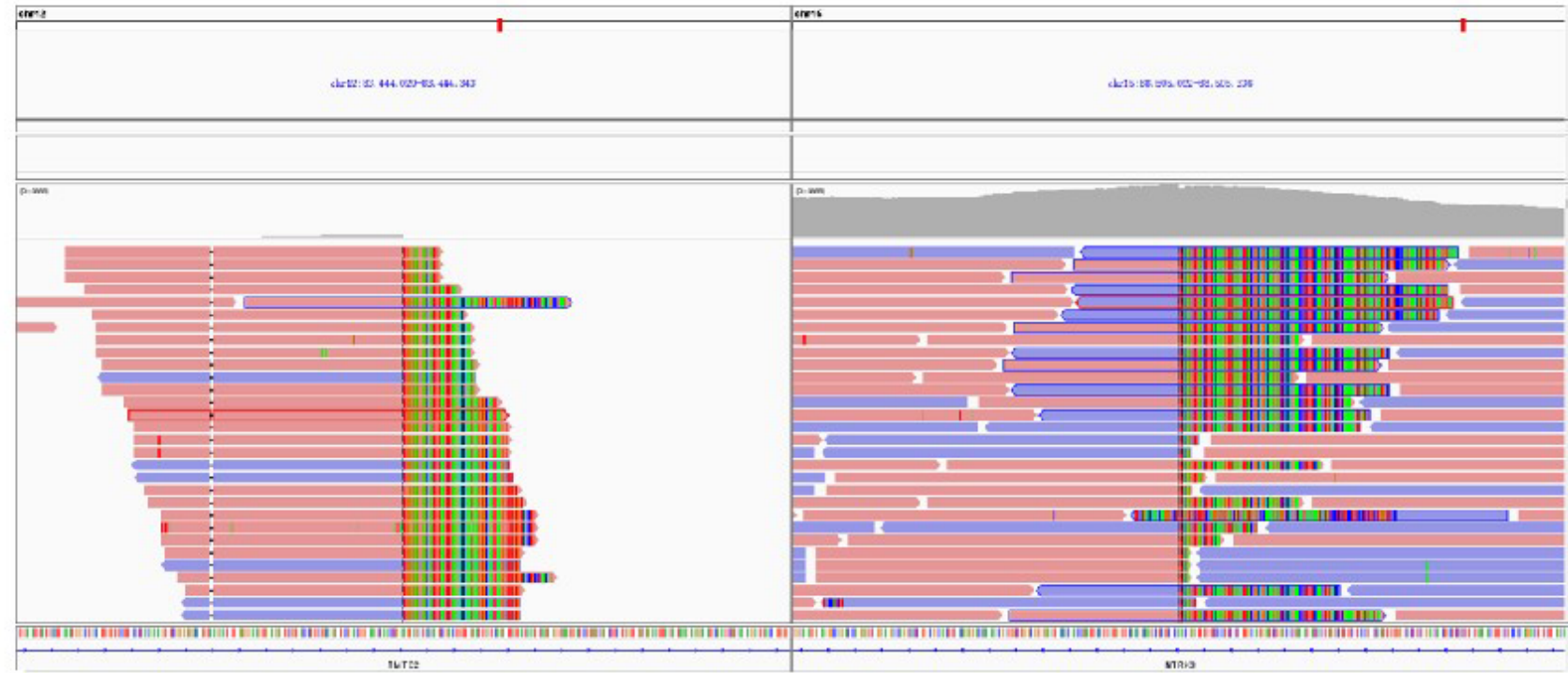

C


\section{Figure 1}

TMTC2-NTRK3 fusion analysis. a Schematic diagram of detailed fusion site of TMTC2 and NTRK3. $b$ NGS results showed a breakpoint of fusion. c TMTC2-NTRK3 fusion was confirmed by FISH and immunohistochemistry of the tumor tissue sample. (Left) Immunohistochemistry staining indicated a strong NTRK3 protein expression. (Right) A split signal was observed in situ hybridization image. 


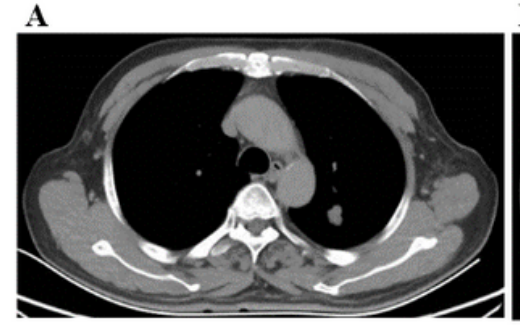

Jun 10,2020

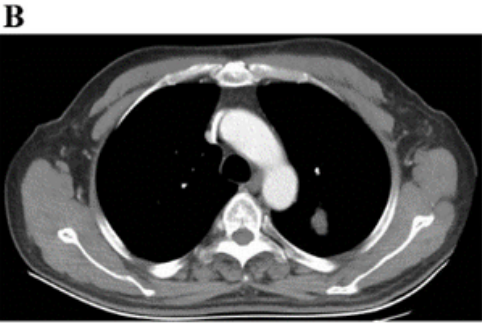

Jul 10, 2020

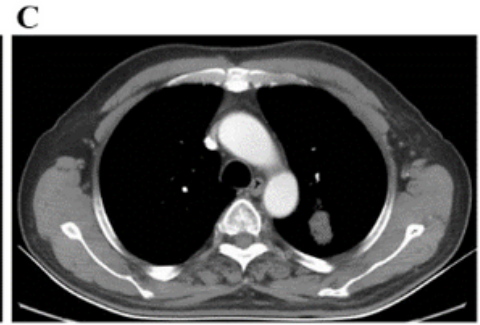

Sep 8, 2020

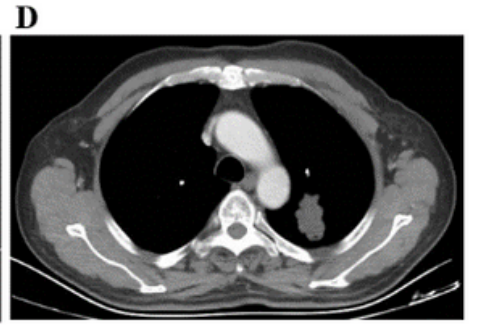

Nov 4, 2020

Figure 2

Representative lung and chest imaging data of the patient until Nov 2020. a Pre-treatment lung CT image. b Lung CT after one month of larotectinib therapy. c,d A Follow-up CT image shows metastases were continuously enlarged than before. 